INPLASY

PROTOCOL

To cite: Xu et al. Efficacy and safety of immune checkpoint inhibitors in neoadjuvant chemotherapy for triplenegative breast cancer: a systematic review and metaanalysis. Inplasy protocol 202180040. doi:

10.37766/inplasy2021.8.0040

Received: 11 August 2021

Published: 11 August 2021

Corresponding author: Jian Zhang

dr_jianzhang@njmu.edu.cn

Author Affiliation:

Department of General

Surgery, the First Affiliated

Hospital of Nanjing Medical

University, Nanjing China.

Support: National Natural Science.

Review Stage at time of this submission: Preliminary searches.

Conflicts of interest:

None declared.

\section{Efficacy and safety of immune checkpoint inhibitors in neoadjuvant chemotherapy for triple-negative breast cancer: a systematic review and meta-analysis}

$$
\text { Xu, Z1; Song, Y²; Xu, D³; Fei, Y4; Tang, J5; Zhang, J6. }
$$

Review question / Objective: We want to conduct a systematic review and meta-analysis of a number of randomized controlled trials (RCTs) to provide the latest evidence on the controversial role of drugs in neoadjuvant chemotherapy in patients with triple negative breast cancer. Condition being studied: Triple negative breast cancer.

Eligibility criteria: The inclusion criteria were: (1) pathological diagnosis of $\mathrm{BC}$ patients with triple negative;(2) a prospective RCT in patients with early breast cancer;(3)RCTs must include an experimental group treated with PD-1 or PD-L1 inhibitors and a control group treated with normal neoadjuvant chemotherapy;(4) pCR data after experimental and control neoadjuvant therapy were provided.

INPLASY registration number: This protocol was registered with the International Platform of Registered Systematic Review and Meta-Analysis Protocols (INPLASY) on 11 August 2021 and was last updated on 11 August 2021 (registration number INPLASY202180040).

\section{INTRODUCTION}

Review question / Objective: We want to conduct a systematic review and metaanalysis of a number of randomized controlled trials (RCTs) to provide the latest evidence on the controversial role of drugs in neoadjuvant chemotherapy in patients with triple negative breast cancer.

Condition being studied: Triple negative breast cancer. 


\section{METHODS}

Participant or population: Patients diagnosed with early-stage triple-negative breast cancer receiving neoadjuvant chemotherapy

Intervention: Triple negative breast cancer Patients receiving neoadjuvant chemotherapy with addition of immune checkpoint inhibitors.

Comparator: Patients with triple-negative breast cancer receiving normal neoadjuvant chemotherapy.

Study designs to be included: We will analyze the effect and side effects of the regimen of adding immune checkpoint inhibitors to neoadjuvant chemotherapy.

Eligibility criteria: The inclusion criteria were: (1) pathological diagnosis of $\mathrm{BC}$ patients with triple negative;(2) a prospective RCT in patients with early breast cancer;(3)RCTs must include an experimental group treated with PD-1 or PD-L1 inhibitors and a control group treated with normal neoadjuvant chemotherapy;(4) pCR data after experimental and control neoadjuvant therapy were provided.

Information sources: Two of the authors searched the PubMed, Web of Science, and Embase databases for prospective clinical trials.

Main outcome(s): Our main research objectives were pathological complete response ( $p C R)$ and adverse events, and subanalysis was performed according to PD-L1 status.

Quality assessment / Risk of bias analysis: The quality of included studies was evaluated by the Newcastle-Ottawa Scale(NOS) by two independent authors. NOS scores which are consisted of 3 parts: comparability (0-2 points), outcome assessment ( $0-3$ points) and selection (0-4 points), $\geq 6$ are viewed as high-quality studies.
Strategy of data synthesis: Two of the authors searched the PubMed, Web of Science, and Embase databases for prospective clinical trials using immunotherapy for neoadjuvant chemotherapy in early TNBC from the inception of each database through publication to January 25, 2021. MeSH terms or text words included "Breast Neoplasm", "Breast Tumor", "Breast Cancer", "Breast Malignant Neoplasm", "Breast Malignant Tumor", "Immune Checkpoint Inhibitors", "PD-1 Inhibitor", "PD-L1 Inhibitor", "nivolumab", "pembrolizumab", "Tislelizumab", "atezolizumab", "durvalumab", "avelumab", "neoadjuvant", "chemotherapy".

Subgroup analysis: We performed a subgroup analysis of PD-L1 status in patients receiving immune checkpoint inhibitors.

Sensitivity analysis: If the included literatures are highly heterogeneous, sensitivity analysis will be performed for them.

Country(ies) involved: China.

Keywords: breast cancer, immune checkpoint inhibitor, neoadjuvant, safety.

Contributions of each author:

Author 1 - Zheng Xu.

Author 2 - Yuxin Song.

Author 3 - Di Xu.

Author 4 - Yinjiao Fei.

Author 5 - Jinhai Tang.

Author 6 - Jian Zhang. 